\title{
Endothelial Aquaporin-1 (AQP1) Expression Is Regulated by Transcription Factor Mef2c
}

\author{
Yong Jiang', , He Liu', Wen-jing Liu', Hai-bin Tong', Chang-jun Chen', Fu-gui Lin', Yan-hang Zhuo', \\ Xiao-zhen Qian', Zeng-bin Wang', Yu Wang', Peng Zhang', and Hong-liang Jia'
}

\begin{abstract}
Aquaporin 1 (AQP1) is expressed in most microvasculature endothelial cells and forms water channels that play major roles in a variety of physiologic processes. This study aimed to delineate the transcriptional regulation of $A Q P 1$ by Mef2c in endothelial cells. Mef2c cooperated with Sp1 to activate human AQP1 transcription by binding to its proximal promoter in human umbilical cord vein endothelial cells (HUVEC). Over-expression of Mef2c, Sp1, or Mef2c/Sp1 increased HUVEC migration and tube-forming ability, which can be abolished AQP1 knockdown. These data indicate that $A Q P 1$ is a direct target of Mef2c in regulating angiogenesis and vasculogenesis of endothelial cells.
\end{abstract}

\section{INTRODUCTION}

Aquaporins (AQP) belong to a family of water or solute channels that are widely expressed in various epithelia and endothelia as well as in other cell types such as epidermis, adipocytes, and skeletal muscle (Papadopoulos and Verkman, 2013; Preston and Agre, 1991). In mammals, there are 13 different AQPs, defined as AQP0 to AQP12 (Verkman and Mitra, 2000). AQP0, 1, 2, 4, 5, and 8 function mainly as water-selective transporters. AQP3, 7, 9, and 10 are aquaglyceroporins that transport both water and glycerol (Verkman et al., 2014). AQPs play important role in regulating physiological functions such as neuroexcitatory processes in central nerve system (Verkman et al., 2011), epidermal proliferation in skin keratinocytes (HaraChikuma and Verkman, 2008), urinary concentration in kidney (Verkman, 2008), and transepithelial fluid secretion in exocrine glands (Tradtrantip et al., 2009). AQPs are also involved in cell migration and vessel formation. Inhibition of AQP dependent angiogenesis impairs tumor growth in a mouse model of melanoma (Saadoun et al., 2005).

AQP1 is specifically and strongly expressed in most microvasculature endothelial cells (Au et al., 2004; Kim and Jung,

\footnotetext{
${ }^{1}$ Medical Examination College, Jilin Medical University, ${ }^{2}$ Life Science Research Center, Beihua University, Jilin, People's Republic of China *Correspondence: jiangyongpost@ sina.com
}

Received 20 August, 2015; revised 25 November, 2015; accepted 26 November, 2015; published online 29 February, 2016

Keywords: angiogenesis, AQP1, Mef2c, migration, vascular tube formation
2011). The increase of AQP1 expression accounts for in myocardial edema during cardiopulmonary bypass (Gao et al., 2012). AQP1 knockdown resulted in high permeability in pulmonary microvasculature (Rutkovskiy et al., 2013). It has been shown that ischemia, hypoxia, and cardioplegia could reduce cardiac AQP1 level (Bondy et al., 1993), where AQP1 is mostly located in microvasculature and t-tubules of myocardial cells (Kim and Jung, 2011; Xiao et al., 2013). Although the distribution and roles of AQP1 in endothelial cells have been established, the regulation of endothelial expression of AQP1 has not been fully understood.

The transcription factor myocyte enhancer factor 2 (MEF2) family proteins bind to a consensus sequence CTA(A/T)4TAG in the promoter region of target genes (Molkentin and Olson, 1996). Mef2c is expressed in endothelial cells (ECs) both in vivo (Lin et al., 1998) and in vitro (Hosking et al., 2001; Maiti et al., 2008) and play a particularly important role in angiogenesis during vascular development. Whole body knockout of Mef2c leads to early embryonic death resulted from cardiac defects while targeted deletion of Mef2c in mice leads to severe vascular defects (Lin et al., 1998). Transcriptome analyses using Mef2-deficient cells identified several downstream genes including c-fos and Arc that function to control synaptic remodeling (Flavell et al., 2008). In the hematopoietic compartment, Mef2c regulates leukemic cell migration and invasionby targeting chemokine ligands (Schwieger et al., 2009), chemokine receptors and matrix metalloproteinase genes. It is still uncertain which componentsare targeted by Mef2c during angiogenesis.

This study aimed to elucidate the regulatory mechanism for AQP1 transcription by Mef2c. The interaction between Mef2c and its binding site was analyzed by DNA affinity precipitation and chromatin immunoprecipitation, cell migration with wound healing assay, and vasculogenesis by tube formation assays. Our data showed that Mef2c cooperated with Sp1 to activate human AQP1 transcription by binding to its proximal promoter in human umbilical cord vein endothelial cells (HUVEC), Overexpression of Mef2c, Sp1, or Mef2c/Sp1 increased HUVEC migration and tube-forming ability, which can be abolished AQP-1 knockdown. The data indicated that Aqp1 is a direct target of Mef2c in endothelial cells and angiogenic and vasculogenic activities of Mef2c.

\section{MATERIAL AND METHODS}

Cell Culture, transient transfection, and luciferase assays Human umbilical cord vein endothelial cells (HUVEC) (ATCC, 
USA) were maintained in DMEM/ high-glucose medium supplemented with $20 \%$ FBS, penicillin-streptomycin (100 U/ml and $100 \mathrm{mg} / \mathrm{ml}$, respectively) (Invitrogen, China), heparin (90 $\mathrm{mg} / \mathrm{ml}$, Sigma, USA), and endothelial cell growth factor (20 $\mathrm{mg} / \mathrm{ml}$, Fisher Scientific, USA) at $37^{\circ} \mathrm{C}$ in a humidified atmosphere with $5 \% \mathrm{CO}_{2}$. One day before transfection, HUVEC cells were seeded into 12 -well plates to allow $50-70 \%$ confluence on the day of transfection. Reporter constructs and expression vectors were transfected using Lipofectamine 2000 (Invitrogen) according to manufacturer's instructions. $50 \mathrm{ng} /$ well vector TKrenilla (Promega, USA) was transfected as internal control. Total DNA was kept at $1.0 \mathrm{mg} /$ well. Corresponding empty vectors were used as standard in different treatment groups. Luciferase activity was measured $48 \mathrm{~h}$ later using dual-luciferase assay kit (Promega) and read on a microtiter plate luminometer (Dynex, USA). All experiments were performed in triplicates. Results were shown as relative luciferase activity and expressed as mean \pm S.E from at least three independent experiments.

\section{Luciferase reporter vectors and expression vectors}

The promoter region of human AQP1 gene was amplified from human genomic DNA with primers listed in Table 1, digested with Bglll and XindllI, and cloned into pGL3-vector which was digested with the same enzymes to generate $1.6 \mathrm{~kb}, 500 \mathrm{bp}$, $260 \mathrm{bp}, 210 \mathrm{bp}, 100 \mathrm{bp}$, and $30 \mathrm{bp}$ constructs. The mutant reporter gene constructs were generated based on pGL3hAQP1-210 with indicated primers (Table 1) using Quickkit (Stratagene). All constructs were confirmed by DNA sequencing. Flag-Mef2c was kindly provided by Dr. Skerjanc (University of Ottawa). Sp1 vector was described elsewhere (Xiao et al., 2013).

\section{Quantitative real-time PCR}

Total RNA from mouse heart or HUVECs was extracted using RNeasy Mini Kit (Qiagen, China) according to manufacturer's instructions. The first strand cDNA was synthesized using a reverse transcription kit from Tiangen Bio (China) according to manufacturer's protocol.

Quantitative real-time PCR was performed with a qPCR kit from TransGen (China) on a Eppendorf Realplex with specified primers (Table. 1). The reaction program was consisted of $95^{\circ} \mathrm{C}$ for $3 \mathrm{~min}$ followed by 40 cycles of $95^{\circ} \mathrm{C} 30 \mathrm{~s}, 55^{\circ} \mathrm{C} 20 \mathrm{~s}$, and $72^{\circ} \mathrm{C} 20 \mathrm{~s}$. GAPDH or $\beta$-actin gene were served as internal control and the relative mRNA levels were calculated by $2^{-\Delta \Delta C t}$.

\section{Western blot}

The total protein from HUVECs was resolved by SDSpolyacrylamide gel electrophoresis before transferred onto a PVDF membrane. The membrane was blocked with $5 \%$ non-fat milk and incubated with primary antibody against AQP1 (USA), Mef2c (Abcam, USA), or Sp1 (Abcam,) followed by incubation with horseradish peroxidase conjugated secondary antibody. An enhanced chemiluminescence (ECL) (Sangon, China) was used to visualize the protein bands. The protein bands were then analyzed by Image $\mathrm{J}$ (National Institutes of Health) software.

\section{DNA affinity precipitation assays (DAPA)}

The extraction of nuclear protein from HUVECs and DNA affinity precipitation assay were performed as described previously (Xiao et al., 2013). The sequences for the oligonucleotides used in DAPA assay were listed in Table 1 with mutated nucleotides in lowercase.

\section{Chromatin immunoprecipitation (CHIP)}

The atria and ventricles of E13 mouse embryos were collected in cold PBS. Mouse heart tissue and HUVEC cells CHIP were performed using an EpiQuik Tissue Chromatin Immunoprecipitation (ChIP) Kit (Epigentek, USA) and am EpiXplore ${ }^{\mathrm{TM}}$ ChIP Assay Kit (Clontech, USA) according to the manufacturers' protocols, respectively. Antibodies used for CHIP were antiMef2c (sc-13266 X, Santa Cruz, USA) or normal goat IgG (control). Chipped DNA samples were subjected to quantitative PCR. Fold enrichment indicated the ratio of CHIPed DNA to the IgG control, normalized for input DNA. Primer sequences were listed in Table 1.

\section{Cell motility assay}

HUVEC cells were seeded in 6-well plates $24 \mathrm{~h}$ before being transfected with indicated plasmids and scratched with a pipette tip $24 \mathrm{~h}$ after transfection. The gap was measured under a microscope immediately after scratching and after $24 \mathrm{~h}$ of culture. The assay was performed three times with 3 replicates each. The relative migration rate was calculated as: relative migration rate $=(d i-d t)_{\exp } /(d i-d t)_{c t r l} \times 100$, where di was the initial gap width, dt was the gap width after $24 \mathrm{~h}$ of culture, exp was individual treatments, and ctrl was the control cell without any treatment.

\section{Vascular tube formation assay}

HUVEC cells were transfected as described $24 \mathrm{~h}$ before the assay. On the day of the assay, $50 \mu \mathrm{l}$ of BD Matrigel was added into 96-well plates and incubated at $37^{\circ} \mathrm{C}$ for $30 \mathrm{~min}$. Tranfected HUVEC cells were trypsinized and adjusted to a concentration of $5 \times 10^{4}$ cells $/ \mathrm{ml}$ with complete culture medium, and $100 \mu \mathrm{l}$ of cells were seeded into each well. The cells were photographed and tubes were counted in 5 random fields (100x) $48 \mathrm{~h}$ later. The assay was performed in three independent experiments with 5 replicates each. The relative tube formation activity $=\mathrm{N}_{\text {exp }} / \mathrm{N}_{\text {ctrl }}$, where $\mathrm{N}_{\exp }$ was the number of vascular tubes of each treatment group and $\mathrm{N}_{\mathrm{Ctrl}}$ was the number of vascular tubes of the control group.

\section{RNA interference against AQP1}

Two pairs of RNA oligonucleotides targeting different regions of AQP1 (Stigliano et al., 2013) and a control pair were customsynthesized by Sangon (China). Double stranded siRNA was transfected into NIH 3T3 cells using Lipofectamine 2000 (Invitrogen, China) according to the manufacturer's instructions. The protein and mRNA level were measured $48 \mathrm{~h}$ later by western blot with specified antibodies and quantitative real-time PCR using ABI Prism 7300 (Applied Biosystems, USA), respectively.

\section{Statistic analysis}

The data was expressed as mean \pm S.E. The differences between groups were analyzed by Student's $t$-test. $P<0.05$ was considered statistically significant.

\section{RESULTS}

Mef2c increased human AQP1 promoter activity

To identify the cis-element important for the transcriptional activation of human AQP1 gene, a serial $5^{\prime}$ truncation of hAQP1 promoter was inserted into PGL3-vector. The luciferase activity was analyzed 2 days after transfection into HUVEC cells. The promoter activity of human AQP1 was almost abrogated in the -30 construct which contained an intact TATA-box whereas all 
Mef2c Regulates Endothelial Aquaporin-1 AQP1 Expression

Yong Jiang et al.

Table 1. The sequences of nucleotide primers used in the study.

\begin{tabular}{|c|c|c|}
\hline & Gene & Oligonucleotide sequence \\
\hline \multirow[t]{14}{*}{ Promoter study } & pAQP1-1.6k_F & GGA AGATCTCCACTCATCCTTGCCCTGCCCC \\
\hline & pAQP1-500_F & GGAAGATCTGTGCCCCCATCCACAGAGCCCT \\
\hline & pAQP1-260_F & GGAAGATCTCCGCCCACCCCACCCACTCC \\
\hline & pAQP1-210_F & GGAAGATCTCCTCCTGCCTGCCTCCTTGGG \\
\hline & pAQP1-100_F & GGAAGATCTACAGCCGTGTCTGCATCCATCCAG \\
\hline & pAQP1_R & CCCAAGCTTAGGGGGCTTGGCCTGAGACCG \\
\hline & pAQP1-30_F & GATCTTATAAATAGGCCCAGCCCAGGCTGTGGCTCAGA \\
\hline & pAQP1-30_R & AGCTTCTGAGCCACAGCCTGGGCTGGGCCTATTTATAA \\
\hline & pAQP1_mSp1 & CTTTGTGCTCCCTATATATCCCGGCCCTAT \\
\hline & & ATAGGGCCGGGATATATAGGGAGCACAAAG \\
\hline & pAQP1_mMef2c & CCGGCCATATAAATATATCCAGCCCAG \\
\hline & & CTGGGCTGGATATATTTATATGGCCGG \\
\hline & pAQP1_mSp1/Mef2c & CTTTGTGCTCCСTATATATCCCGGCCATATAAATATATCCAGCCCAG \\
\hline & & CTGGGCTGGATATATTTATATGGCCGGGATATATAGGGAGCACAAAG \\
\hline \multirow[t]{12}{*}{ q-PCR } & $\mathrm{mmAQP1}$ & GGGCAGTAATATCACTGTCTT \\
\hline & & GTGGGTCCCTCACTTTCA \\
\hline & hAQP1 & TCTCAGGCATCACCTCCTCC \\
\hline & & CGAGTTCACACCATCAGCCA \\
\hline & mmMef2c & САССТАCATAACATGCCGCC \\
\hline & & AGGCTTTGAGTAGAAGGCAGG \\
\hline & hMef2c & TGGAGAAGCACTTCAACGCT \\
\hline & & CCTGATGAAGGAAGACCCGA \\
\hline & $\mathrm{mm} \beta$-actin & GGTCATCACTATTGGCAACG \\
\hline & & ACGGATGTCAACGTCACACT \\
\hline & hGAPDH & GCACCGTCAAGGCTGAGAAC \\
\hline & & TGGTGAAGACGCCAGTGGA \\
\hline \multirow[t]{6}{*}{ siRNA } & siAQP1-1S & GGGUGGAGAUGAAGCCCAAdTdT \\
\hline & siAQP1-1A & UUGGGCUUCAUCUCCACCCdTdT \\
\hline & siAQP1-2S & UGGAGAUGAAGCCCAAAUAdTdT \\
\hline & siAQP1-2A & UAUUUGGGCUUCAUCUCCAdTdT \\
\hline & siControl-S & UGGAGAAGGCCAACUAGGGdTdT \\
\hline & siControl-A & CCCUAGUUGGCCUUCUCCAdTdT \\
\hline \multirow[t]{4}{*}{ DAPA assay } & bio-AQP1_F & biotin-TGTGCTCCCCCCGCCCCCCGGCCCTATAAATAGGCCCAGCC \\
\hline & AQP1_R & GGCTGGGCCTATTTATAGGGCCGGGGGGCGGGGGGAGCACA \\
\hline & bio-mAQP1_F & biotin-TGTGCTCCaCaCGaCCaCCGaCCaTATAAATAaGaCCAGCC \\
\hline & mAQP1_R & GGCTGGtCtTATTTATAtGGtCGGtGGtCGtGtGGAGCACA \\
\hline \multirow[t]{8}{*}{ CHIP-qPCR } & hCHIP1_F & TGTCTCTGCCACTCT \\
\hline & hCHIP1_R & GACTTGAGGAAAGAAGCA \\
\hline & hCHIP2_F & AGGTCTGTGTGGTGT \\
\hline & hCHIP2_R & TCAATTCCСTCTGAGA \\
\hline & mmCHIP1_F & TGGAAGATGGAATTCGT \\
\hline & mmCHIP1_R & СТСТАСCСAGTTCACT \\
\hline & mmCHIP2_F & GGCCTTCCTCCCTTT \\
\hline & mmCHIP2_R & ATGCCTGGTGCTCAA \\
\hline
\end{tabular}

h, human; m, mutant; mm, mouse

other constructs had consistently high promoter activities (Fig. 1A). A Mef2 binding site was identified assessing hAQP1 proximal promoter sequence, which was conserved among human, mouse, and rat (Fig. 1B). When cotransfecting with Mef2c expression vector, the luciferase activity of hAQP1 $(-1000 \mathrm{~kb})$ was 2.4-fold higher than cotransfected with control empty vector (Fig. $1 \mathrm{C)}$.
Mef2c cooperated with Sp1 in activating human AQP1 transcription

To confirm the role of Mef2 binding site in human AQP1 transcription, the Mef2 site and the adjacent Sp1 binding site were mutated individually or simultaneously followed by luciferase assays. Mutating the Mef2, Sp1, or both Mef2 and Sp1 sites caused $50 \%, 70 \%$, and $90 \%$ reduction of AQP1 promoter ac- 
A

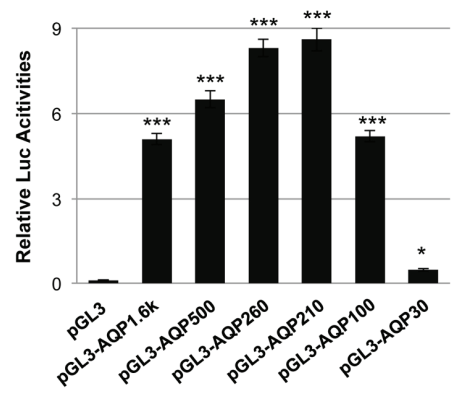

C

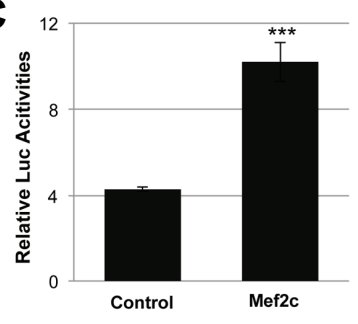

Sp1-binding site

Mef2c-binding site

B

Human GCGGGCCAGGAGCGAAGAGAGGCCTTCCTCCCTTTGTGCTCCCCCCgCCCCCCGGCDCTATAAATAGGCCCAGCCCAGGCTGTGGCTCA Mouse GCGGGCCAGGAGCA.AGGAGAGGCCTTCCTCCCTT TGTGCTCCCCCC---ACCCAGCDCTATAAATAGGCCCAGCCOAGGCTGT GGCTCAG

Rat GCGGGCCAGGAGCAAGGAGAGGCCTTCCTCCCTT TGTGCTCCCCCC---ACCCA GCDCTATAAATAGGCCCAGCCCAGGCTGT GGCT CAG
Fig. 1. Identification of transcription factorsimportant for human Aqp1 expression. (A) Human AQP1 promoter $5^{\prime}$ deletion luciferase report constructs were transfected into HUVEC cells and luciferase activities were measured $48 \mathrm{~h}$ later. (B) The alignment of the proximal region of human, mouse, and rat AQP1 promoters. The grey shaded region indicated Sp1-binding (GC-rich) site, the boxed area indicated the Mef2c-binding site which overlapped with TATA box. (C) Human AQP1 reporter gene (pAQP1-210) was co-transfected with either pcDNA3.1 or Mef2c expression vector into HUVEC cells followed by luciferase activity assay 48 $\mathrm{h}$ later.
A

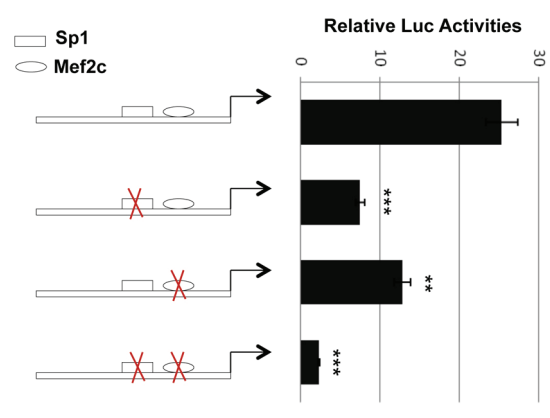

C

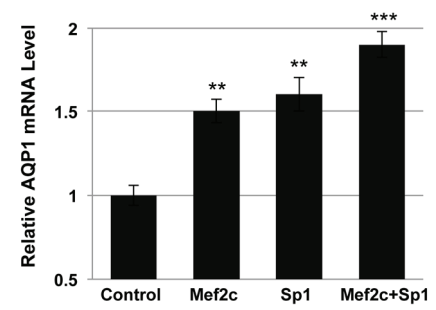

B

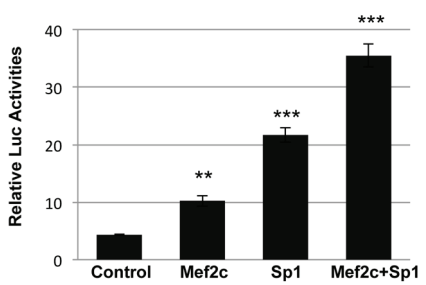

D

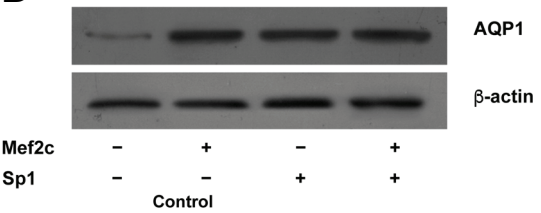

E

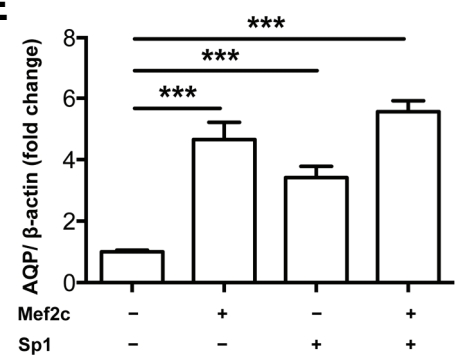

Fig. 2. Mef2c activates the transcription of human Aqp1 gene. (A). Wild type pAQP1100 or Mef2c and/ or Sp1-binding site mutant constructs were transfected into HUVEC cells and luciferase activities were measured 2 days later. (B) pAQP1-100 construct was cotransfected with Mef2c, $\mathrm{Sp1}$, or both and luciferase activities were measured $48 \mathrm{~h}$ post-transfection. HUVEC cells were transfected with empty vector, Mef2c, Sp1, or both, (C) AQP1 mRNA and $(D, E)$ protein levels were analyzed $48 \mathrm{~h}$ later. tivity (Table 1, pAQP1_mSp1, pAQP1_mMef2, pAQP1_mSp1/ Mef2 and Fig. 2A). On the other hand, cotransfection of Mef2c, Sp1, or Mef2c/Sp1 increased human AQP1 promoter activity 2.4, 5.0, and 8.3 folds, respectively (Fig. 2B). The AQP1 mRNA levels in HUVEC cells transfected with Mef2c, Sp1, and Mef2c/Sp1 expression vectors were $50 \%, 60 \%$, and $90 \%$ higher than that of control cells (Fig. 2C). The AQP1 protein levels HUVEC cells were also significantly increased by over expression of Mef2c and/ or Sp1 (Figs. 2D and 2E).

\section{Mef2c bound to human AQP1 proximal promoter}

Using biotin labeled polynucleotides corresponding to the human AQP1 promoter region containing Sp1 and Mef2c binding sites, the Mef2c and Sp1 proteins were precipitated from
HUVEC nuclear proteins by wild type polynucleotide but not by the polynucleotide with mutated Mef2c and Sp1-binding sites (Figs. 3A and 3B). Mef2c specific antibodies strongly enriched the chromatin fragments containing Mef2c binding sites but not other regions in the AQP1 promoter without a Mef2c binding site in either HUVEC cells (Fig. 3C) or mouse hearts (Fig. 3D).

\section{Mef2c regulated HUVEC migration through AQP1}

Knockdown AQP1 expression reduced the migrating ability of HUVEC cells by $85 \%$ (Fig. 4A). On the other hand, overexpression of Mef2c, Sp1, or Mef2c/Sp1 increased HUVEC migration by $84 \%, 92 \%$, and $154 \%$, respectively. Knockdown AQP1 completely abrogated the increased motility of HUVEC cells resulted from Mef2c/Sp1 over-expression (Fig. 4B). 
A

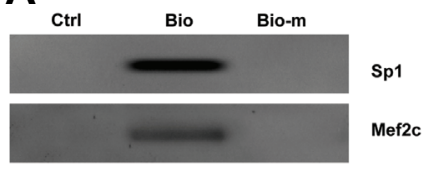

C

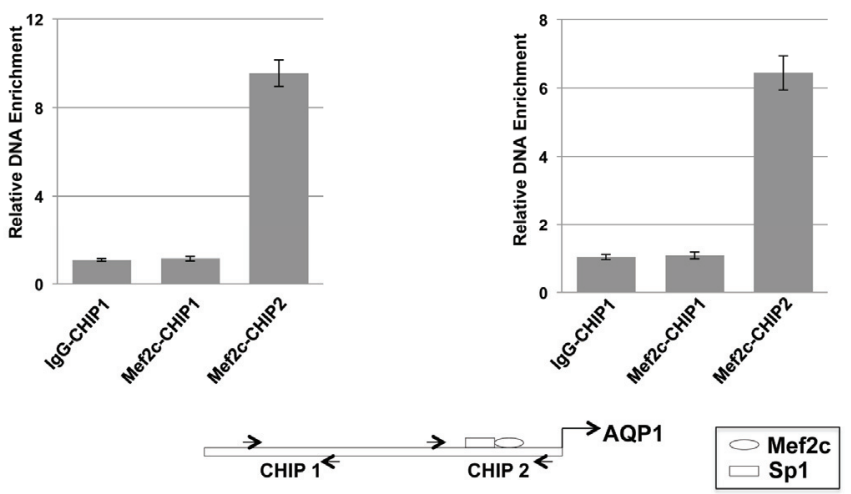

Fig. 3. Mef2c and $S p 1$ bind to the Mef2 and $S p 1$ sites in human AQP1 promoter. (A, B) 5 ' biotin labeled nucleotides corresponding to the Mef2 and Sp1 sites or their mutants of human AQP1 promoter were incubated with HUVEC cell nuclear extracts and precipitated with TetralinkTMavidin resin. The DNA-protein complexes were resolved in SDSPAGE gel and immunoblotted with antibodies against Mef2c and Sp1. HUVEC cells (C) and embryonic mouse heart tissues (D) were chromatin immunoprecipitated with antibody against Mef2c or normal IgG and the resultant DNA samples were analyzed by qPCR with primers encompassing regions with or without Mef2 binding site.

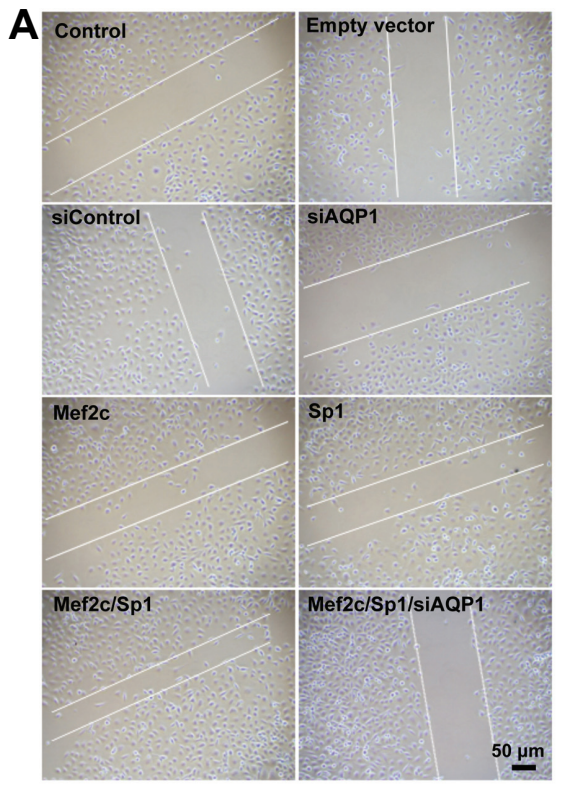

Fig. 4. Mef2c promotes HUVEC migration through AQP1. (A) HUVEC cells transfected with control empty vectors, siAQP1, Mef2c, Sp1, Mef2c/Sp1, or Mef2c/Sp1/siAQP1 were scratched with a blue tip $24 \mathrm{~h}$ after transfection. The gap distance was measured after scratch and $24 \mathrm{~h}$ later. (B) The relative migration distance of cells with different treatment was calculated against untreated cells.
Mef2c increased vascular tube formation of HUVEC cells Over-expression of Mef2c, Sp1, and Mef2c/Sp1 significantly increased in vitro tube-forming ability of HUVEC cells by 2 to 4 folds, and such increase was abolished by knockdown AQP1 expression (Fig. 5A). Knockdown AQP1 expression alone in HUVEC cells reduced tube number by more than $50 \%$ (Fig. 5B).

\section{DISCUSSION}

Mef2c activates human AQP1 transcription in endothelial cells with cooperation of Sp1 through the specific binding sites in the proximal promoter region. Disrupting either MEF2 or adjacent Sp1 binding site or both significantly reduce AQP1 promoter activities. Mef2c controlls HUVEC cells migration and tube forming through regulation of AQP1 expression.

AQP1 promotes angiogenesis by increasing the motility of endothelial cells (Saadoun et al., 2005; Stigliano et al., 2013). AQP1-null endothelial cells migrates slower more than $50 \%$ compared with wild-type cells, which is recovered by ectopic AQP1 expression (Saadoun et al., 2005). The lack of AQP1 impaires tumor angiogenesis and tumor growth due to increased necrosis (Stigliano et al., 2013), and causes abnormal microvascular structure and reduces vascular density in breast cancer (Hara-Chikuma and Verkman, 2006). The present data showed that knockdown AQP1 level reduced both migration and vascular tube formation of endothelial cells in basal and 


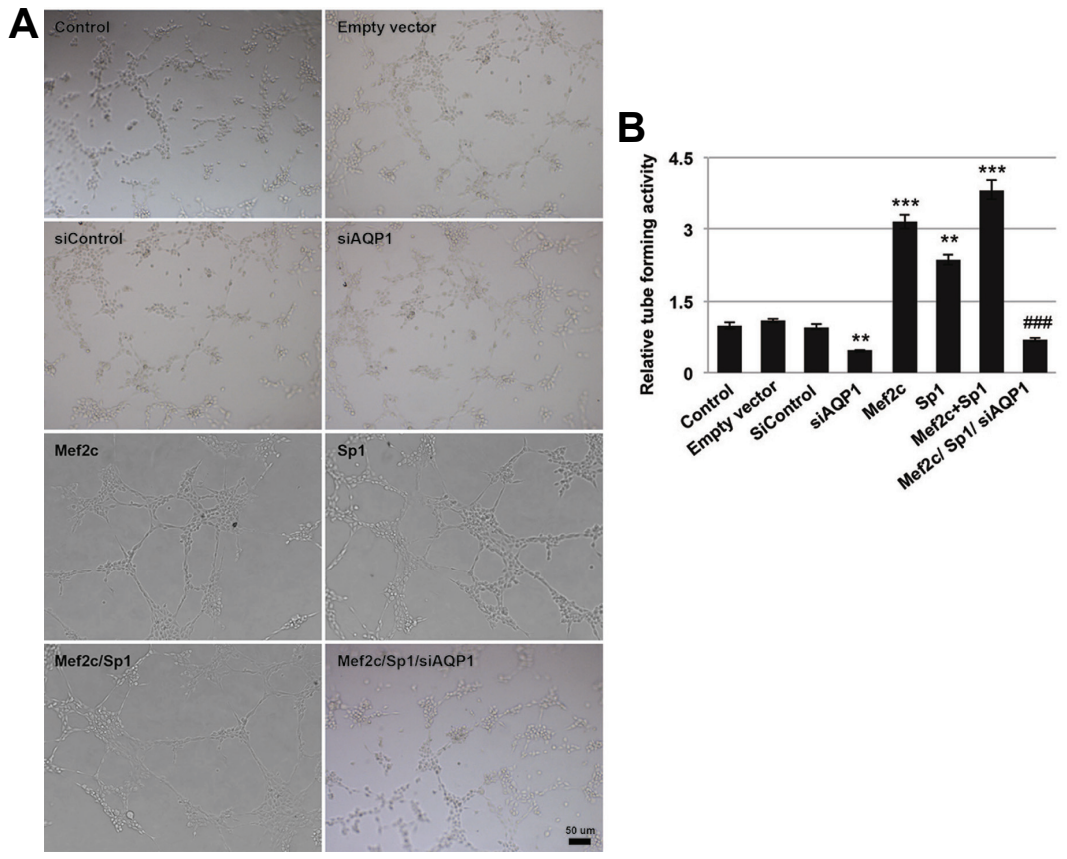

Fig. 5. Mef2c promotes HUVEC cell tube formation through AQP1. (A) HUVEC cells transfected with control empty vectors, SiAQP1, Mef2c, Sp1, Mef2c/Sp1, or Mef2c/Sp1/siAQP1 were seeded on Matrigel and incubated for 24 $\mathrm{h}$ before being photographed and analysis. (B) The relative tube-forming activity of cells with different treatment was calculated against untreated cells.

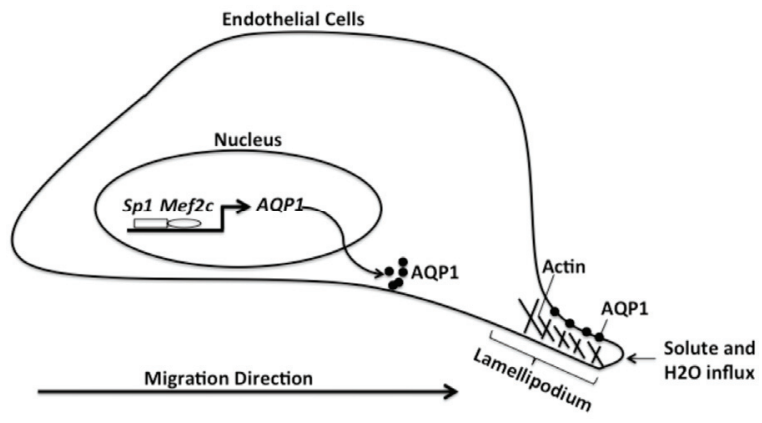

Fig. 6. The regulation of AQP1 by Mef2c in endothelial cells during vasculogenesis and angiogenesis

Mef2c-overexpression background.

Mef2c has been shown to be critical for vasculogenesis and angiogenesis during vascular development and mediate vascular endothelial growth factor (Maiti et al., 2008). Mef2c deficiency causes severe abnormalities of vascular development including failed maturation of extra embryonic vasculature, severe malformation of embryonic vasculature, and malformed endocardium ( $\mathrm{Bi}$ et al., 1999). Mef2 protein is significantly reduced in myotonic dystrophy type 1 heart accompanied by a change of expression profile of both microRNAs and mRNAs, which could be restored by exogenous Mef2c (Esteva-Font et al., 2014). Deletion of Mef2c from endothelial cells represses vessel loss in oxygen-induced retinopathy and significantly increased revascularization (Kalsotra et al., 2014).

The activity of Mef2c during vascular development is modulated by homeodomain interacting protein kinase through direct interaction in a transforming growth factor (TGF)- $\beta$-TAK1dependent manner (Xu et al., 2012). Sry-related HMG box gene Sox18 and Mef2c interacts with each other in endothelial cells to direct the transcription of genes involved in vascular development (Shang et al., 2013). Mef2c also interacts with SOX10 to potentiate its own transcription in melanocyte development (Hosking et al., 2001). In the present study, Mef2c functionally interacted with Sp1 to activate the transcription of AQP1 gene, which partially conferred the angiogenic activity of Mef2c.

All together, these data expand understanding of the physiological role of AQP1 except for its water transport function. The regulation of AQP1 by Mef2c in endothelial cells during vasculogenesis and angiogenesis are summarized in Fig. 6. It has been demonstrated that interactions of aquaporin and cytoskeletal elements or ion transporters at the tip of a lamellipodium drive the influx of water across the cell membrane (Saadoun et al., 2005). Solute and water influx then increases local hydrostatic pressure to create space for actin polymerization, which might contribute to aquaporin-dependent cell human endothelial migration. Earlier study by Abreu-Rodriguez et al. (2011) show that up-regulation of AQP1 by hypoxia may result in increased membrane water permeability and account for edema associated in tumor mircroenvrioment. HIF1- $\alpha$ and other transcription factors are identified to regulate AQP1 promotor activity. Our findings suggest that different transcription factors may involve in regulation of AQP1 in normal cells and in diseases and thepossibility to consider tissue specific AQP1 targeting forvarious pathophysiological conditions.

\section{ACKNOWLEDGMENTS}

The author would like to thank Dr. Yan Wang (Chi Biotechnology and University of Pennsylvania) for critical reading. This work was supported by the Youth Research Fund Project of Science and Technology Department of Jilin Province (20140520006JH), National Natural Science Foundation of China (31100566), the Training Program for Outstanding Young Talents in Jilin (20156425), Chunmiao Cultivation Program for University Talents in Jilin Province (2013-352), Jilin Provincial Health Department Project. 


\section{REFERENCES}

Abreu-Rodriguez, I., Sanchez Silva, R., Martins, A.P., Soveral, G., Toledo-Aral, J.J., Lopez-Barneo, J., and Echevarria, M. (2011). Functional and transcriptional induction of aquaporin-1 gene by hypoxia; analysis of promoter and role of Hif-1alpha. PLoS One 6 , e28385.

Au, C.G., Cooper, S.T., Lo, H.P., Compton, A.G., Yang, N., Wintour, E.M., North, K.N., and Winlaw, D.S. (2004). Expression of aquaporin 1 in human cardiac and skeletal muscle. J. Mol. Cell Cardiol. 36, 655-662.

Bi, W., Drake, C.J., and Schwarz, J.J. (1999). The transcription factor MEF2C-null mouse exhibits complex vascular malformations and reduced cardiac expression of angiopoietin 1 and VEGF. Dev. Biol. 211, 255-267.

Bondy, C., Chin, E., Smith, B.L., Preston, G.M., and Agre, P. (1993). Developmental gene expression and tissue distribution of the CHIP28 water-channel protein. Proc. Natl. Acad. Sci. USA 90, 4500-4504.

Esteva-Font, C., Jin, B.J., and Verkman, A.S. (2014). Aquaporin-1 gene deletion reduces breast tumor growth and lung metastasis in tumor-producing MMTV-PyVT mice. FASEB J. 28, 1446-1453.

Flavell, S.W., Kim, T.K., Gray, J.M., Harmin, D.A., Hemberg, M., Hong, E.J., Markenscoff-Papadimitriou, E., Bear, D.M., and Greenberg, M.E. (2008). Genome-wide analysis of MEF2 transcriptional program reveals synaptic target genes and neuronal activity-dependent polyadenylation site selection. Neuron 60, 1022-1038.

Gao, C., Tang, J., Li, R., and Huan, J. (2012). Specific inhibition of AQP1 water channels in human pulmonary microvascular endothelial cells by small interfering RNAs. J. Trauma Acute Care Surg. 72, 150-161.

Hara-Chikuma, M., and Verkman, A.S. (2006). Aquaporin-1 facilitates epithelial cell migration in kidney proximal tubule. J. Am. Soc. Nephrol. 17, 39-45.

Hara-Chikuma, M., and Verkman, A.S. (2008). Roles of aquaporin3 in the epidermis. J. Invest Dermatol. 128, 2145-2151.

Hosking, B.M., Wang, S.C., Chen, S.L., Penning, S., Koopman, P., and Muscat, G.E. (2001). SOX18 directly interacts with MEF2C in endothelial cells. Biochem. Biophys. Res. Commun. 287, 493500.

Kalsotra, A., Singh, R.K., Gurha, P., Ward, A.J., Creighton, C.J., and Cooper, T.A. (2014). The Mef2 transcription network is disrupted in myotonic dystrophy heart tissue, dramatically altering miRNA and mRNA expression. Cell Rep. 6, 336-345.

Kim, J., and Jung, Y. (2011). Different expressions of AQP1, AQP4, eNOS, and VEGF proteins in ischemic versus non-ischemic cerebropathy in rats: potential roles of AQP1 and eNOS in hydrocephalic and vasogenic edema formation. Anat. Cell Biol. 44, 295-303.

Lin, Q., Lu, J., Yanagisawa, H., Webb, R., Lyons, G.E., Richardson, J.A., and Olson, E.N. (1998). Requirement of the MADS-box transcription factor MEF2C for vascular development. Development 125, 4565-4574.

Maiti, D., Xu, Z., and Duh, E.J. (2008). Vascular endothelial growth factor induces MEF2C and MEF2-dependent activity in endothelial cells. Invest Ophthalmol. Vis. Sci. 49, 3640-3648.

Molkentin, J.D., and Olson, E.N. (1996). Combinatorial control of muscle development by basic helix-loop-helix and MADS-box transcription factors. Proc. Natl. Acad. Sci. USA 93, 9366-9373.

Papadopoulos, M.C., and Verkman, A.S. (2013). Aquaporin water channels in the nervous system. Nat. Rev. Neurosci. 14, 265-277.

Potthoff, M.J., and Olson, E.N. (2007). MEF2: a central regulator of diverse developmental programs. Development 134, 4131-4140.

Preston, G.M., and Agre, P. (1991). Isolation of the cDNA for erythrocyte integral membrane protein of 28 kilodaltons: member of an ancient channel family. Proc. Natl. Acad. Sci. USA 88, 11110-11114.

Rutkovskiy, A., Bliksoen, M., Hillestad, V., Amin, M., Czibik, G. Valen, G., Vaage, J., Amiry-Moghaddam, M., and Stenslokken, K.O. (2013). Aquaporin-1 in cardiac endothelial cells is downregulated in ischemia, hypoxia and cardioplegia. J. Mol. Cell Cardiol. 56, 22-33.

Saadoun, S., Papadopoulos, M.C., Hara-Chikuma, M., and Verkman, A.S. (2005). Impairment of angiogenesis and cell migration by targeted aquaporin-1 gene disruption. Nature 434, 786-792.

Schwieger, M., Schuler, A., Forster, M., Engelmann, A., Arnold, M.A., Delwel, R., Valk, P.J., Lohler, J., Slany, R.K., Olson, E.N., et al. (2009). Homing and invasiveness of MLL/ENL leukemic cells is regulated by MEF2C. Blood 114, 2476-2488.

Shang, Y., Doan, C.N., Arnold, T.D., Lee, S., Tang, A.A., Reichardt, L.F., and Huang, E.J. (2013). Transcriptional corepressors HIPK1 and HIPK2 control angiogenesis via TGF-beta-TAK1dependent mechanism. PLoS Biol. 11, e1001527.

Stigliano, C., Aryal, S., de Tullio, M.D., Nicchia, G.P., Pascazio, G. Svelto, M., and Decuzzi, P. (2013). siRNA-chitosan complexes in poly(lactic-co-glycolic acid) nanoparticles for the silencing of aquaporin-1 in cancer cells. Mol. Pharm. 10, 3186-3194.

Tradtrantip, L., Tajima, M., Li, L., and Verkman, A.S. (2009). Aquaporin water channels in transepithelial fluid transport. J. Med. Invest 56, 179-184.

Verkman, A.S. (2008). Dissecting the roles of aquaporins in renal pathophysiology using transgenic mice. Semin. Nephrol. 28, 217226.

Verkman, A.S., and Mitra, A.K. (2000). Structure and function of aquaporin water channels. Am J. Physiol. Renal. Physiol. 278, F13-28.

Verkman, A.S., Ratelade, J., Rossi, A., Zhang, H., and Tradtrantip, L. (2011). Aquaporin-4: orthogonal array assembly, CNS functions, and role in neuromyelitis optica. Acta Pharmacol. Sin. 32, 702-710.

Verkman, A.S., Anderson, M.O., and Papadopoulos, M.C. (2014). Aquaporins: important but elusive drug targets. Nat. Rev. Drug Discov. 13, 259-277.

Xiao, J., Zhou, Y., Lai, H., Lei, S., Chi, L.H., and Mo, X. (2013) Transcription factor NF-Y is a functional regulator of the transcription of core clock gene Bmal1. J. Biol. Chem. 288, 31930-31936.

Xu, Z., Gong, J., Maiti, D., Vong, L., Wu, L., Schwarz, J.J., and Duh, E.J. (2012). MEF2C ablation in endothelial cells reduces retinal vessel loss and suppresses pathologic retinal neovascularization in oxygen-induced retinopathy. Am. J. Pathol. 180, 2548-2560. 